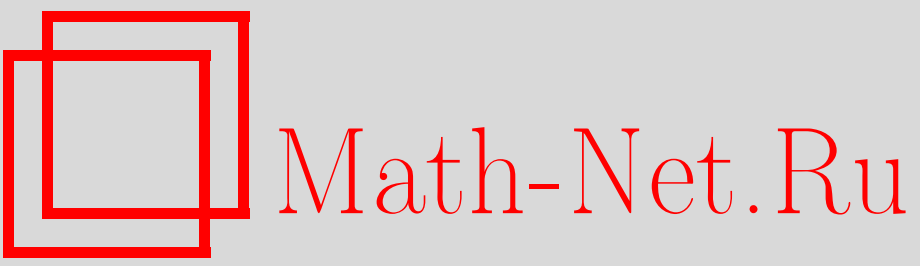

Л. С. Кузьменков, С. Г. Максимов, О функциях распределения в квантовой механике и функциях Вигнера, ТМФ, 2002, том 131, номер 2, 231-243

DOI: https://doi.org/10.4213/tmf327

Использование Общероссийского математического портала Math-Net.Ru подразумевает, что вы прочитали и согласны с пользовательским соглашением

http://www.mathnet.ru/rus/agreement

Параметры загрузки:

IP: 54.196 .121 .252

26 апреля 2023 г., 04:59:41 
ТЕОРЕТИЧЕСКАЯ

И МАТЕМАТИЧЕСКАЯ

ФИЗИКА

Том 131, № 2

май, 2002

(C) 2002 г. $\quad$ Л. С. Кузьменков* , С. Г. Максимов ${ }^{\dagger}$

\title{
О ФУНКЦИЯХ РАСПРЕДЕЛЕНИЯ В КВАНТОВОЙ МЕХАНИКЕ И ФУНКЦИЯХ ВИГНЕРА
}

\begin{abstract}
Сформулирована и решена задача об отыскании функции распределения $F(\mathbf{r}, \mathbf{p}, t)$, приводящая в результате вычисления статистических средних к тем же локальным значениям числа частиц, импульса и энергии, что и квантовая механика. Метод основан на квантово-механическом определении плотности вероятности, не ограниченном числом частиц системы. Найденная функция распределения совпадает с функцией Вигнера лишь для пространственно-однородных систем. Получены цепочка уравнений Боголюбова, уравнение Лиувилля для квантовых функций распределения при любом числе частиц в системе, квантовое кинетическое уравнение с самосогласованным электромагнитным полем, общее выражение для тензора диэлектрической проницаемости электронной компоненты плазмы. Этот тензор наряду с известными физическими эффектами, определяющими дисперсию продольных и поперечных волн в плазме, содержит вклад обменных кулоновских корреляций, существенный для плотных систем.
\end{abstract}

\section{1. ВВЕДЕНИЕ}

Динамику системы $N$ взаимодействуюших частиц можно исследовать как математическими методами классической физики, так и методами квантовой теории. Представление динамики частиц как динамики уединенных $\delta$-образных волновых пакетов может быть получено путем явного учета в уравнениях движения определения точечной частицы, а классическая микроскопическая гидродинамика может быть получена как динамика пространственных распределений $\sum_{i=1}^{N} m_{i} \delta\left(\mathbf{r}-\mathbf{r}_{i}(t)\right)$ всех частиц системы. При больших $N$ может быть достигнуто макроскопическое представление путем дополнительного определения плотности числа частиц как нормированной на число частиц плотности вероятности нахождения частицы в физически бесконечно малой окрестности $\Delta(\mathbf{r})$ каждой точки пространства.

В квантовой механике динамика той же системы определяется волновой функцией в конфигурационном пространстве, причем квантово-механическое определение плотности вероятности также является дополнительным для уравнения Шредингера. Мик-

\footnotetext{
* Московский государственный университет, Москва, Россия. E-mail: 1sk@nabla.phys.msu.su

†Instituto Tecnológico de Morelia, México. E-mail: ccpenagos@yahoo.com
} 
роскопическую квантовую гидродинамику (МКГ) системы можно получить аналогично путем явного включения в динамику квантово-механического определения плотности вероятности. Основные положения этой программы и некоторые приложения изложены, в частности, в работах [1]-[5].

Более детальное классическое описание физических процессов в системе $N$ частиц основано на изучении лиш части частиц из окрестности $\Delta(\mathbf{r})$, принадлежащих в определенный момент времени окрестности $\Delta(\mathbf{p})$ импульсного пространства. Плотность числа частищ в шестимерном объеме $\Delta(\mathbf{r}) \Delta(\mathbf{p})$ есть одночастичная функция распределения системы. Поэтому математически бесконечно малым $\Delta(\mathbf{r})$ и $\Delta(\mathbf{p})$ соответствует микроскопическая функция распределения $\sum_{i=1}^{N} \delta\left(\mathbf{r}-\mathbf{r}_{i}(t)\right) \delta\left(\mathbf{p}-\mathbf{p}_{i}(t)\right)$. Уравнение для этой функции будет содержать двухчастичную микроскопическую функцию распределения. Полная иерархия микроскопических распределений при любом числе частиц в системе удовлетворяет цепочке уравнений Боголюбова и уравнению Лиувилля [6]. При этом гидродинамические уравнения могут быть получены из статистических при помощи обычной процедуры интегрирования по импульсам. Ясно, что такому представлению должно соответствовать квантовое микроскопическое статистическое представление динамики, из которого фундаментальные уравнения МКГ должны появляться при помоши интегрирования по импульсам. Качественные отличия квантовых микроскопических статистических уравнений от классических будут диктоваться принципом неопределенности. Следует ожидать, что производные по координатам от квантовых распределений не будут входить в уравнения одновременно с производными по импульсам.

В качестве исходного положения для построения квантовой функции распределения может быть принято правило соответствия Вейля между классическими динамическими функциями и эрмитовыми операторами. Классическими аналогами функций распределения в этом случае будут функции Вигнера [7], [8]. Однако плотность кинетической энергии частиц, вычисленная на основе одночастичной функции Вигнера, оказывается не совпадающей с плотностью кинетической энергии в МКГ (см. ниже). Уравнения МКГ, в свою очередь, являются прямым следствием квантовой механики. При их выводе использовались лишь уравнение Шредингера и вероятностная интерпретация волновой функции. Поэтому возможности построения функции распределения в действительности ограничены следуюшими условиями: а) концентрация частиц при любом числе частиц в системе может быть вычислена путем интегрирования квантовой одночастичной функции распределения по импульсам; б) квантовый ток, найденный по правилам квантовой механики, может быть вычислен как статистическое среднее от импульса р с одночастичной функцией распределения; в) плотность кинетической энергии в квантовой механике может быть вычислена как статистическое среднее от величины $\mathbf{p}^{2} / 2 m$ с одночастичной функцией распределения. Эти условия будут использованы ниже для построения микроскопических квантовых функций распределения, соответствующих аналогичным классическим функциям. Многочастичные функции распределения при таком подходе будут автоматически появляться в каждом уравнении цепочки Боголюбова. 


\section{2. КВАНТОВЫЕ МИКРОСКОПИЧЕСКИЕ РАСПРЕДЕЛЕНИЯ}

При указанных условиях в квантовой теории функции $\sum_{i=1}^{N} \delta\left(\mathbf{r}-\mathbf{r}_{i}(t)\right) \delta\left(\mathbf{p}-\mathbf{p}_{i}(t)\right)$ соответствует оператор

$$
\hat{f}(\mathbf{r}, \mathbf{p})=\sum_{i=1}^{N} \hat{n}_{i}(\mathbf{p}) \hat{n}_{i}(\mathbf{r})
$$

где $N$ - число частиш в системе, $\hat{n}_{i}(\mathbf{r})$ и $\hat{n}_{i}(\mathbf{p})$ - операторы плотности вероятности для $i$-й частицы иметь соответственно координату $\mathbf{r}$ и импульс $\mathbf{p}$. Поэтому проблема построения квантовой микроскопической функции распределения сводится к приведению к общему представлению операторов $\hat{n}_{i}(\mathbf{r})$ и $\hat{n}_{i}(\mathbf{p})$ в формуле $(1)$.

В r-представлении

$$
\begin{aligned}
\left\langle\mathbf{r}_{i}\left|\hat{n}_{i}(\mathbf{p})\right| \mathbf{r}_{i}^{\prime}\right\rangle & =\int d \mathbf{p}_{i} \int d \mathbf{p}_{i}^{\prime}\left\langle\mathbf{r}_{i} \mid \mathbf{p}_{i}\right\rangle\left\langle\mathbf{p}_{i}\left|\hat{n}_{i}(\mathbf{p})\right| \mathbf{p}_{i}^{\prime}\right\rangle\left\langle\mathbf{p}_{i}^{\prime} \mid \mathbf{r}_{i}^{\prime}\right\rangle= \\
& =\frac{1}{(2 \pi \hbar)^{3}} e^{\frac{i}{\hbar} \mathbf{p}\left(\mathbf{r}_{i}-\mathbf{r}_{i}^{\prime}\right)}
\end{aligned}
$$

и матричный элемент оператора (1) примет вид

$$
\begin{aligned}
f\left(\mathbf{r}, \mathbf{p} \mid R, R^{\prime}\right) & =\sum_{i=1}^{N} \int d \mathbf{r}_{i}^{\prime \prime}\left\langle\mathbf{r}_{i}\left|\hat{n}_{i}(\mathbf{p})\right| \mathbf{r}_{i}^{\prime \prime}\right\rangle\left\langle\mathbf{r}_{i}^{\prime \prime}\left|\hat{n}_{i}(\mathbf{r})\right| \mathbf{r}_{i}^{\prime}\right\rangle \prod_{\substack{k=1 \\
k \neq i}}^{N} \delta\left(\mathbf{r}_{k}-\mathbf{r}_{k}^{\prime}\right)= \\
& =\sum_{i=1}^{N} \delta\left(\mathbf{r}-\mathbf{r}_{i}^{\prime}\right) \frac{1}{(2 \pi \hbar)^{3}} e^{\frac{i}{\hbar} \mathbf{p}\left(\mathbf{r}_{i}-\mathbf{r}_{i}^{\prime}\right)} \prod_{\substack{k=1 \\
k \neq i}}^{N} \delta\left(\mathbf{r}_{k}-\mathbf{r}_{k}^{\prime}\right),
\end{aligned}
$$

где $R=\left\{\mathbf{r}_{1}, \ldots, \mathbf{r}_{N}\right\}$. Такой оператор, очевидно, сушествует для любой системы $N$ взаимодействующих частиц. Что касается оператора $\hat{f}(\mathbf{r}, \mathbf{v})$, где $\mathbf{v}-$ скорость, то он существует только при равном нулю магнитном поле В. В соответствии с правилом построения одночастичных аддитивных операторов в представлении вторичного квантования [9] мы получаем

$$
\hat{f}(\mathbf{r}, \mathbf{p}, t)=\frac{1}{(2 \pi \hbar)^{3}} a_{\mathbf{p}}^{+}(t) e^{-\frac{i}{\hbar} \mathbf{p r}} \Psi(\mathbf{r}, t)=\int \frac{d \mathbf{q}}{(2 \pi \hbar)^{3}} e^{-\frac{i}{\hbar} \mathbf{p q}} \widehat{\mathcal{R}}(\mathbf{r}-\mathbf{q} ; \mathbf{r}, t),
$$

где $\widehat{\mathcal{R}}\left(\mathbf{r} ; \mathbf{r}^{\prime}, t\right)=\Psi^{+}(\mathbf{r}, t) \Psi\left(\mathbf{r}^{\prime}, t\right)$ - одночастичньй статистический оператор [10].

Операторы (3), (4) не являются эрмитовыми. Эрмитова операторная функция распределения $\widehat{F}(\mathbf{r}, \mathbf{p})$ может быть представлена линейной комбинацией двух эрмитовых операторов:

$$
\widehat{F}_{\{a, b\}}(\mathbf{r}, \mathbf{p})=a \frac{\hat{f}(\mathbf{r}, \mathbf{p})+\hat{f}^{+}(\mathbf{r}, \mathbf{p})}{2}+b \frac{\hat{f}(\mathbf{r}, \mathbf{p})-\hat{f}^{+}(\mathbf{r}, \mathbf{p})}{2 i} .
$$


Плотности числа частиц $\hat{n}(\mathbf{r}, t)$, квантово-механического тока $\hat{\mathbf{j}}(\mathbf{r}, t)$ и кинетической энергии $\hat{\varepsilon}_{\text {kin }}(\mathbf{r}, t)$ по условию равны:

$$
\begin{gathered}
\int d \mathbf{p} \widehat{F}(\mathbf{r}, \mathbf{p}, t)=\hat{n}(\mathbf{r}, t), \\
\int d \mathbf{p}\left(\mathbf{p}-\frac{e}{c} \mathbf{A}(\mathbf{r}, t)\right) \widehat{F}(\mathbf{r}, \mathbf{p}, t)=\hat{\mathbf{j}}(\mathbf{r}, t), \\
\int d \mathbf{p} \frac{1}{2 m}\left(\mathbf{p}-\frac{e}{c} \mathbf{A}(\mathbf{r}, t)\right)^{2} \widehat{F}(\mathbf{r}, \mathbf{p}, t)=\hat{\varepsilon}_{\mathbf{k i n}}(\mathbf{r}, t),
\end{gathered}
$$

где [1]-[5], [9]

$$
\begin{gathered}
\hat{n}(\mathbf{r}, t)=\Psi^{+}(\mathbf{r}, t) \Psi(\mathbf{r}, t), \\
\hat{\mathbf{j}}(\mathbf{r}, t)=\frac{1}{2}\left(\Psi^{+}(\mathbf{r}, t)(\mathbf{D} \Psi)(\mathbf{r}, t)+(\mathbf{D} \Psi)^{+}(\mathbf{r}, t) \Psi(\mathbf{r}, t)\right), \\
\hat{\varepsilon}_{\mathrm{kin}}(\mathbf{r}, t)=\frac{1}{4 m}\left(\Psi^{+}(\mathbf{r}, t)\left(\mathbf{D}^{2} \Psi\right)(\mathbf{r}, t)+\left(\mathbf{D}^{2} \Psi\right)^{+}(\mathbf{r}, t) \Psi(\mathbf{r}, t)\right), \\
\mathbf{D}=\frac{\hbar}{i} \nabla-\frac{e}{c} \mathbf{A}(\mathbf{r}, t) .
\end{gathered}
$$

С другой стороны, из формул (4) и (5) находим

$$
\begin{gathered}
\int d \mathbf{p} \widehat{F}_{\{a, b\}}(\mathbf{r}, \mathbf{p}, t)=a \hat{n} \\
\int d \mathbf{p}\left(\mathbf{p}-\frac{e}{c} \mathbf{A}(\mathbf{r}, t)\right) \widehat{F}_{\{a, b\}}(\mathbf{r}, \mathbf{p}, t)=a \hat{\mathbf{j}}+b \frac{\hbar}{2} \nabla \hat{n} \\
\int d \mathbf{p} \frac{1}{2 m}\left(\mathbf{p}-\frac{e}{c} \mathbf{A}(\mathbf{r}, t)\right)^{2} \widehat{F}_{\{a, b\}}(\mathbf{r}, \mathbf{p}, t)=a \hat{\varepsilon}_{\text {kin }}+b \frac{\hbar}{2 m}\left(\nabla \hat{\mathbf{j}}+\hat{n} \frac{e}{c}(\nabla \mathbf{A})\right) .
\end{gathered}
$$

Из (6) и (8) следует, что $a=1, b=0$. При этом квантово-механические плотности (7) могут быть вычислены одновременно и как статистические средние с операторной функцией распределения $\widehat{F}(\mathbf{r}, \mathbf{p}, t)=\left(\hat{f}+\hat{f}^{+}\right) / 2$. Функция $\widehat{F}_{q}(\mathbf{r}, \mathbf{p}, t)=\left(\hat{f}-\hat{f}^{+}\right) / 2 i$, как видно из (8), приводит к статистическим средним градиентов квантово-механических плотностей (7) с множителем $\hbar$ и, следовательно, имеет чисто квантовую природу. Можно показать, что для локально-равновесных систем частищ между $\widehat{F}$ и $\widehat{F}_{q}$ имеет место соотношение

$$
\widehat{F}_{q}(\mathbf{r}, \mathbf{p}, t)=\frac{3 \hbar}{2} \nabla\left\{\frac{\mathbf{p}-\mathcal{P}(\mathbf{r}, t)}{|\mathbf{p}-\mathcal{P}(\mathbf{r}, t)|^{2}} \widehat{F}(\mathbf{r}, \mathbf{p}, t)\right\},
$$

где $\mathcal{P}(\mathbf{r}, t)$ - произвольное векторное поле. Отсюда видно, в частности, что для пространственно-однородных распределений $\widehat{F}_{q}=0$.

Наличие двух эрмитовых операторных функций $\widehat{F}$ и $\widehat{F}_{q}$ в микроскопической теории связано с комплексным характером волновой функции, и в дальнейшем удобно рассматривать неэрмитову операторную функцию $\hat{f}(\mathbf{r}, \mathbf{p}, t)$. 
Нетрудно убедиться в сушествовании функциональной связи функции распределения $\hat{f}(\mathbf{r}, \mathbf{p}, t)$ с функцией Вигнера $\hat{f}_{\mathrm{W}}(\mathbf{r}, \mathbf{p}, t)[7],[8]$

$$
\hat{f}_{\mathrm{W}}(\mathbf{r}, \mathbf{p}, t)=\int \frac{d \mathbf{q}}{(2 \pi \hbar)^{3}} e^{\frac{i}{\hbar} \mathbf{p q}} \widehat{\mathcal{R}}\left(\mathbf{r}+\frac{\mathbf{q}}{2} ; \mathbf{r}-\frac{\mathbf{q}}{2}, t\right) .
$$

Эта связь имеет вид

$$
\hat{f}(\mathbf{r}, \mathbf{p}, t)=\int d \mathbf{p}^{\prime} \int \frac{d \mathbf{q}}{(2 \pi \hbar)^{3}} e^{-\frac{i}{\hbar}\left(\mathbf{p}-\mathbf{p}^{\prime}\right) \mathbf{q}} \hat{f}_{\mathrm{W}}\left(\mathbf{r}-\frac{\mathbf{q}}{2}, \mathbf{p}^{\prime}, t\right),
$$

откуда видно, что обе операторные функции совпадают лишш для пространственно-однородных систем. В общем случае неоднородных систем функции $\hat{f}_{\text {и }} \hat{f}_{\mathrm{W}}$ приводят к разным значениям плотности кинетической энергии системы частиц. Используя функцию Вигнера, получаем

$$
\int d \mathbf{p} \frac{\left(\mathbf{p}-\frac{e}{c} \mathbf{A}\right)^{2}}{2 m} \hat{f}_{\mathrm{W}}(\mathbf{r}, \mathbf{p}, t)=\hat{\varepsilon}_{\mathrm{kin}}(\mathbf{r}, t)+\frac{\hbar^{2}}{8 m} \Delta \hat{n}(\mathbf{r}, t),
$$

что не совпадает с известным выражением (7) для кинетической энергии $\hat{\varepsilon}_{\mathrm{kin}}(\mathbf{r}, t)$ в случае скалярного поля [9].

\section{3. СИСТЕМА УРАВНЕНИЙ ДЛЯ МИКРОСКОПИЧЕСКИХ РАСПРЕДЕЛЕНИЙ}

Установим уравнение для одночастичной операторной функции $\hat{f}(\mathbf{r}, \mathbf{p}, t)$. Для этого заметим, что статистический оператор для системы $N$ кулоновских частиц во внешшем электромагнитном поле удовлетворяет уравнению

$$
\begin{aligned}
& i \hbar \frac{\partial \widehat{\mathcal{R}}(\mathbf{r}-\mathbf{q} ; \mathbf{r}, t)}{\partial t}=\frac{1}{2 m}\left\{\left(\frac{\hbar}{i}\left(\frac{\partial}{\partial \mathbf{r}}+\frac{\partial}{\partial \mathbf{q}}\right)-\frac{e}{c} \mathbf{A}(\mathbf{r}, t)\right)^{2}-\right. \\
& \left.\quad-\left(\frac{\hbar}{i} \frac{\partial}{\partial \mathbf{q}}-\frac{e}{c} \mathbf{A}(\mathbf{r}-\mathbf{q}, t)\right)^{2}\right\} \widehat{\mathcal{R}}(\mathbf{r}-\mathbf{q} ; \mathbf{r}, t)+e\left(\hat{L}_{\mathbf{q}} \varphi(\mathbf{r}, t)\right) \widehat{\mathcal{R}}(\mathbf{r}-\mathbf{q} ; \mathbf{r}, t)+ \\
& \quad+\int d \mathbf{r}^{\prime}\left(\hat{L}_{\mathbf{q}} \Phi\left(\mathbf{r}-\mathbf{r}^{\prime}\right)\right) \widehat{\mathcal{R}}_{2}\left(\mathbf{r}-\mathbf{q}, \mathbf{r}^{\prime} ; \mathbf{r}^{\prime}, \mathbf{r}, t\right),
\end{aligned}
$$

где $\Phi\left(\mathbf{r}-\mathbf{r}^{\prime}\right)=e^{2} /\left|\mathbf{r}-\mathbf{r}^{\prime}\right|$, а оператор сдвига $\hat{L}_{\mathbf{q}}$ определен как

$$
\hat{L}_{\mathbf{q}} \varphi(\mathbf{r}, t)=\varphi(\mathbf{r}, t)-\varphi(\mathbf{r}-\mathbf{q}, t) .
$$

Используя (12), находим

$$
\left(\frac{\partial}{\partial t}+\frac{1}{m}\left(\mathbf{p}-\frac{e}{c} \mathbf{A}(\mathbf{r}, t)\right) \frac{\partial}{\partial \mathbf{r}}-\frac{i \hbar}{2 m} \Delta\right) \hat{f}(\mathbf{r}, \mathbf{p}, t)-
$$




$$
\begin{aligned}
& -\int \frac{d \mathbf{k}}{(2 \pi)^{3}} e^{i \mathbf{k r}}\left\{\frac{1}{m}\left(\mathbf{p}+\frac{\hbar \mathbf{k}}{2}\right) \frac{e}{c} \mathbf{A}(\mathbf{k}, t)-\right. \\
& \left.-\frac{1}{2 m}\left(\frac{e}{c}\right)^{2} \int \frac{d \mathbf{k}^{\prime}}{(2 \pi)^{3}} \mathbf{A}\left(\mathbf{k}^{\prime}, t\right) \mathbf{A}\left(\mathbf{k}-\mathbf{k}^{\prime}, t\right)-e \varphi(\mathbf{k}, t)\right\} \times \\
& \times \frac{i}{\hbar}(\hat{f}(\mathbf{r}, \mathbf{p}, t)-\hat{f}(\mathbf{r}, \mathbf{p}+\hbar \mathbf{k}, t))= \\
= & -\int d \mathbf{r}^{\prime} \int d \mathbf{p}^{\prime} \int \frac{d \mathbf{k}}{(2 \pi)^{3}} \Phi(\mathbf{k}) e^{i \mathbf{k}\left(\mathbf{r}-\mathbf{r}^{\prime}\right)} \times \\
& \times \frac{i}{\hbar}\left(\hat{f_{2}}\left(\mathbf{r}, \mathbf{p} ; \mathbf{r}^{\prime}, \mathbf{p}^{\prime}, t\right)-\hat{f}_{2}\left(\mathbf{r}, \mathbf{p}+\hbar \mathbf{k} ; \mathbf{r}^{\prime}, \mathbf{p}^{\prime}, t\right)\right) .
\end{aligned}
$$

Уравнение (13) для одночастичной функции, так же как и уравнение для функции Вигнера [8], не содержит производных по импульсам одночастичной и двухчастичной функций. Вместо производных по импульсам в уравнение (13) входят лишш конечные разности, что является следствием принципа неопределенности Гайзенберга. Кроме того, уравнение (13) содержит оператор Лапласа $\hat{f}(\mathbf{r}, \mathbf{p}, t)$ с коэффициентом $\hbar$, отсутствующий в аналогичном уравнении для функции Вигнера. Это означает, что даже в отсутствие взаимодействия между частицами уравнение (13) сохраняет квантовые свойства системы, и граничные условия для функции распределения должны соответствовать граничным условиям для волновой функции.

Соответствие между уравнением (13) и аналогичным классическим уравнением цепочки Боголюбова можно установить путем разложения конечных разностей вида $\hat{f}(\mathbf{r}, \mathbf{p}, t)-\hat{f}(\mathbf{r}, \mathbf{p}+\hbar \mathbf{k}, t)$ в ряд по $\hbar$ с последующим переходом к квазиклассическому пределу. Далее, так как в уравнении (13) двухчастичная микроскопическая операторная функция распределения

$$
\hat{f}_{2}\left(\mathbf{r}, \mathbf{p} ; \mathbf{r}^{\prime}, \mathbf{p}^{\prime}, t\right)=\int \frac{d \mathbf{q} d \mathbf{q}^{\prime}}{(2 \pi \hbar)^{6}} e^{-\frac{i}{\hbar}\left(\mathbf{p q}+\mathbf{p}^{\prime} \mathbf{q}^{\prime}\right)} \widehat{\mathcal{R}}_{2}\left(\mathbf{r}-\mathbf{q}, \mathbf{r}^{\prime}-\mathbf{q}^{\prime} ; \mathbf{r}^{\prime}, \mathbf{r}, t\right)
$$

появляется автоматически, уравнение для нее может быть получено так же, как и уравнение (13). Оно будет содержать трехчастичную функцию распределения. В общем случае уравнение для $s$-частичной операторной функции

$$
\begin{aligned}
\hat{f}_{s}\left(\mathbf{r}_{1}, \mathbf{p}_{1} ; \ldots ; \mathbf{r}_{s}, \mathbf{p}_{s}, t\right)= & \int\left(\prod_{k=1}^{s} \frac{d \mathbf{q}_{k}}{(2 \pi \hbar)^{3}}\right) \exp \left\{-\frac{i}{\hbar} \sum_{k=1}^{s} \mathbf{p}_{k} \mathbf{q}_{k}\right\} \times \\
& \times \widehat{\mathcal{R}}_{s}\left(\mathbf{r}_{1}-\mathbf{q}_{1}, \ldots, \mathbf{r}_{s}-\mathbf{q}_{s} ; \mathbf{r}_{s}, \ldots, \mathbf{r}_{1}, t\right)
\end{aligned}
$$

может быть найдено путем дифференцирования выражения (15) по времени и учета уравнения для $s$-частичного статистического оператора, содержашегося в определении (15):

$$
i \hbar \frac{\partial}{\partial t} \widehat{\mathcal{R}}_{s}\left(R_{s}-Q_{s} ; R_{s}, t\right)=\sum_{i=1}^{s}\left\{\frac{1}{2 m}\left(\frac{\hbar}{i}\left(\frac{\partial}{\partial \mathbf{r}_{i}}+\frac{\partial}{\partial \mathbf{q}_{i}}\right)-\frac{e}{c} \mathbf{A}\left(\mathbf{r}_{i}, t\right)\right)^{2}-\right.
$$




$$
\begin{aligned}
& \left.-\frac{1}{2 m}\left(\frac{\hbar}{i} \frac{\partial}{\partial \mathbf{q}_{i}}-\frac{e}{c} \mathbf{A}\left(\mathbf{r}_{i}-\mathbf{q}_{i}, t\right)\right)^{2}+e\left(\hat{L}_{\mathbf{q}_{i}} \varphi\left(\mathbf{r}_{i}, t\right)\right)\right\} \widehat{\mathcal{R}}_{s}\left(R_{s}-Q_{s} ; R_{s}, t\right)+ \\
& +\frac{1}{2} \sum_{\substack{i, j=1 \\
i \neq j}}^{s}\left(\hat{L}_{\mathbf{q}_{i}-\mathbf{q}_{j}} \Phi\left(\mathbf{r}_{i}-\mathbf{r}_{j}\right)\right) \widehat{\mathcal{R}}_{s}\left(R_{s}-Q_{s} ; R_{s}, t\right)+ \\
& +\sum_{i=1}^{s} \int d \mathbf{r}_{s+1}\left(\hat{L}_{\mathbf{q}_{i}} \Phi\left(\mathbf{r}_{i}-\mathbf{r}_{s+1}\right)\right) \widehat{\mathcal{R}}_{s+1}\left(R_{s}-Q_{s}, \mathbf{r}_{s+1} ; \mathbf{r}_{s+1}, R_{s}, t\right),
\end{aligned}
$$

где $R_{s}=\left\{\mathbf{r}_{1}, \ldots, \mathbf{r}_{s}\right\}$ и $Q_{s}=\left\{\mathbf{q}_{1}, \ldots, \mathbf{q}_{s}\right\}$. Таким образом, мы приходим к цепочке уравнений Боголюбова для квантовых микроскопических операторных функций распределения:

$$
\begin{aligned}
i \hbar \frac{\partial}{\partial t} \hat{f}_{s} & \left(x_{1}, \ldots, x_{s}, t\right)= \\
= & \sum_{i=1}^{s}\left\{-\frac{\hbar^{2}}{2 m} \Delta_{i}+\frac{1}{m}\left(\mathbf{p}_{i}-\frac{e}{c} \mathbf{A}\left(\mathbf{r}_{i}, t\right)\right) \frac{\hbar}{i} \frac{\partial}{\partial \mathbf{r}_{i}}\right\} \hat{f}_{s}\left(x_{1}, \ldots, x_{s}, t\right)+ \\
& +\sum_{i=1}^{s} \int \frac{d \mathbf{k}}{(2 \pi)^{3}} e^{i \mathbf{k r}_{i}}\left\{-\frac{1}{m}\left(\mathbf{p}_{i}+\frac{\hbar \mathbf{k}}{2}\right) \frac{e}{c} \mathbf{A}(\mathbf{k}, t)+\right. \\
& \left.+\frac{1}{2 m} \int \frac{d \mathbf{k}^{\prime}}{(2 \pi)^{3}}\left(\frac{e}{c}\right)^{2} \mathbf{A}\left(\mathbf{k}^{\prime}, t\right) \mathbf{A}\left(\mathbf{k}-\mathbf{k}^{\prime}, t\right)+e \varphi(\mathbf{k}, t)\right\} \times \\
& \times\left(\hat{f}_{s}\left(x_{1}, \ldots, x_{s}, t\right)-\hat{f}_{s}\left(x_{1}, \ldots ; \mathbf{r}_{i}, \mathbf{p}_{i}+\hbar \mathbf{k} ; \ldots, x_{s}, t\right)\right)+ \\
& +\frac{1}{2} \sum_{i, j=1}^{s} \int \frac{d \mathbf{k}}{(2 \pi)^{3}} e^{i \mathbf{k}\left(\mathbf{r}_{i}-\mathbf{r}_{j}\right)} \Phi(\mathbf{k})\left(\hat{f}_{s}\left(x_{1}, \ldots, x_{s}, t\right)-\right. \\
& \left.-\hat{f}_{s}\left(x_{1}, \ldots ; \mathbf{r}_{i}, \mathbf{p}_{i}+\hbar \mathbf{k} ; \ldots ; \mathbf{r}_{j}, \mathbf{p}_{j}-\hbar \mathbf{k} ; \ldots, x_{s}, t\right)\right)+ \\
& +\sum_{i=1}^{s} \int d \mathbf{r}_{s+1} \int d \mathbf{p}_{s+1} \int \frac{d \mathbf{k}}{(2 \pi)^{3}} e^{i \mathbf{k}\left(\mathbf{r}_{i}-\mathbf{r}_{s+1}\right)} \Phi(\mathbf{k}) \times \\
& \times\left(\hat{f}_{s+1}\left(x_{1}, \ldots, x_{s+1}, t\right)-\hat{f}_{s+1}\left(x_{1}, \ldots ; \mathbf{r}_{i}, \mathbf{p}_{i}+\hbar \mathbf{k} ; \ldots, x_{s+1}, t\right)\right)
\end{aligned}
$$

где $x_{i}=\left\{\mathbf{r}_{i}, \mathbf{p}_{i}\right\}, s=\overline{1, N}$. При $s=N \hat{f}_{s+1}=0$, и уравнение (17) представляет собой уравнение Лиувилля для квантового статистического микроскопического оператора в пространстве $\left\{\mathbf{r}_{1}, \mathbf{p}_{1} ; \ldots ; \mathbf{r}_{N}, \mathbf{p}_{N}\right\}$. Это уравнение справедливо при любом $N$.

\section{4. КВАНТОВОЕ МИКРОСКОПИЧЕСКОЕ КИНЕТИЧЕСКОЕ УРАВНЕНИЕ С САМОСОГЛАСОВАННЫМ ПОЛЕМ}

Усредним уравнение для одночастичной операторной функции распределения (13) по квантовым состояниям системы $|C\rangle$, где $|C\rangle$ - волновая функция в пространстве Фока. Тем самым мы получим уравнение для числовой функции $f(\mathbf{r}, \mathbf{p}, t)=\langle C|\hat{f}(\mathbf{r}, \mathbf{p}, t)| C\rangle$. 
Для выделения самосогласованного поля воспользуемся известным групповым разложением $[8]$ для системы фермионов. Тогда

$$
\begin{aligned}
& f_{2}\left(x_{1}, x_{2}, t\right)=f\left(x_{1}\right) f\left(x_{2}\right)- \\
& \quad-e^{-\frac{i}{\hbar}\left(\mathbf{p}_{1}-\mathbf{p}_{2}\right)\left(\mathbf{r}_{1}-\mathbf{r}_{2}\right)} f\left(\mathbf{r}_{1}, \mathbf{p}_{2}, t\right) f\left(\mathbf{r}_{2}, \mathbf{p}_{1}, t\right)+g_{2}\left(x_{1}, x_{2}, t\right),
\end{aligned}
$$

и становится возможным представление уравнения для одночастичной функции $f(\mathbf{r}, \mathbf{p}, t)$ в виде системы уравнений:

$$
\begin{aligned}
& \left(\frac{\partial}{\partial t}+\frac{1}{m}\left(\mathbf{p}-\frac{e}{c} \mathbf{A}(\mathbf{r}, t)\right) \frac{\partial}{\partial \mathbf{r}}-\frac{i \hbar}{2 m} \Delta\right) f(\mathbf{r}, \mathbf{p}, t)- \\
& -\int \frac{d \mathbf{k}}{(2 \pi)^{3}} e^{i \mathbf{k r}}\left\{\frac{1}{m}\left(\mathbf{p}+\frac{\hbar \mathbf{k}}{2}\right) \frac{e}{c} \mathbf{A}(\mathbf{k}, t)-\right. \\
& \left.-\frac{1}{2 m}\left(\frac{e}{c}\right)^{2} \int \frac{d \mathbf{k}^{\prime}}{(2 \pi)^{3}} \mathbf{A}\left(\mathbf{k}^{\prime}, t\right) \mathbf{A}\left(\mathbf{k}-\mathbf{k}^{\prime}, t\right)-e \varphi(\mathbf{k}, t)\right\} \times \\
& \quad \times \frac{i}{\hbar}(f(\mathbf{r}, \mathbf{p}, t)-f(\mathbf{r}, \mathbf{p}+\hbar \mathbf{k}, t))= \\
& =\int d \mathbf{r}^{\prime} \int d \mathbf{p}^{\prime} \int \frac{d \mathbf{k}}{(2 \pi)^{3}} \Phi(\mathbf{k}) e^{-\frac{i}{\hbar}\left(\mathbf{p}-\mathbf{p}^{\prime}\right)\left(\mathbf{r}-\mathbf{r}^{\prime}\right)} f\left(\mathbf{r}, \mathbf{p}^{\prime}, t\right) \times \\
& \quad \times \frac{i}{\hbar}\left(e^{i \mathbf{k}\left(\mathbf{r}-\mathbf{r}^{\prime}\right)} f\left(\mathbf{r}^{\prime}, \mathbf{p}, t\right)-f\left(\mathbf{r}^{\prime}, \mathbf{p}+\hbar \mathbf{k}, t\right)\right)- \\
& -\int d \mathbf{r}^{\prime} \int d \mathbf{p}^{\prime} \int \frac{d \mathbf{k}}{(2 \pi)^{3}} \Phi(\mathbf{k}) e^{i \mathbf{k}\left(\mathbf{r}-\mathbf{r}^{\prime}\right) \times} \\
& \quad \times \frac{i}{\hbar}\left(g_{2}\left(\mathbf{r}, \mathbf{p} ; \mathbf{r}^{\prime}, \mathbf{p}^{\prime}, t\right)-g_{2}\left(\mathbf{r}, \mathbf{p}+\hbar \mathbf{k} ; \mathbf{r}^{\prime}, \mathbf{p}^{\prime}, t\right)\right), \\
& \operatorname{div} \mathbf{E}=4 \pi e \int d \mathbf{p} f(\mathbf{r}, \mathbf{p}, t)+4 \pi \rho_{\mathrm{ext}}(\mathbf{r}, t), \\
& \operatorname{rot} \mathbf{B}=\frac{1}{c} \frac{\partial \mathbf{E}}{\partial t}+\frac{4 \pi e}{c} \int d \mathbf{p} \frac{1}{m}\left(\mathbf{p}-\frac{e}{c} \mathbf{A}(\mathbf{r}, t)\right) \times \\
& \times \frac{1}{2}\left(f(\mathbf{r}, \mathbf{p}, t)+f^{*}(\mathbf{r}, \mathbf{p}, t)\right)+\frac{4 \pi}{c} \mathbf{j}_{\mathrm{ext}}(\mathbf{r}, t), \\
& \operatorname{div} \mathbf{B}=0, \\
& \operatorname{rot} \mathbf{E}=-\frac{1}{c} \frac{\partial \mathbf{B}}{\partial t} .
\end{aligned}
$$

Здесь через А и $\varphi$ обозначены потенциалы полного электромагнитного поля, включающего самосогласованное и внешнее поля. В уравнениях (19) мы учли также самосогласованное поперечное поле на основании изложенного в работе [4] принципа соответствия с классической кинетической теорией. В уравнение Власова, как известно, внешнее и самосогласованное электромагнитные поля входят равноправно. 


\section{5. ТЕНЗОР ДИЭЛЕКТРИЧЕСКОЙ ПРОНИЦАЕМОСТИ КВАНТОВОЙ ПЛАЗМЫ}

Найдем тензоры проводимости и диэлектрической проницаемости для электрон-ионной плазмы, при этом будем рассматривать ионы в качестве компенсируюшего фона. Такая задача имеет самостоятельный интерес, так как обшие формулы для этих тензоров составляют основу электродинамики сред с пространственной и временной дисперсиями. Вместе с тем вывод закона Ома для таких сред из уравнений (19) может попутно служить демонстрацией того факта, что потенциалы А и $\varphi$ входят в (19) в виде комбинаций, соответствующих напряженностям полей. В уравнении для функции Вигнера это условие не выполняется; функция Вигнера не является калибровочно-инвариантной [11].

При $\mathbf{A}=0$ и $\varphi=0$

$$
f(\mathbf{r}, \mathbf{p}, t)=f_{0}(\mathbf{r}, \mathbf{p})=F_{0}(\mathbf{p}) .
$$

При малых $\mathbf{A}$ и $\varphi$

$$
f(\mathbf{r}, \mathbf{p}, t)=F_{0}(\mathbf{p})+\delta f(\mathbf{r}, \mathbf{p}, t),
$$

где $\delta f(\mathbf{r}, \mathbf{p}, t)$ - малая поправка. Пренебрегая в (19) членами второго порядка малости и переходя к представлению Фурье, находим

$$
\begin{gathered}
\int d \mathbf{p}^{\prime}\left(\delta\left(\mathbf{p}-\mathbf{p}^{\prime}\right)-\mathcal{H}\left(\mathbf{k}, \mathbf{p}+\frac{\hbar \mathbf{k}}{2}, \omega\right) \Phi\left(\frac{\mathbf{p}-\mathbf{p}^{\prime}}{\hbar}\right)\right) \delta f\left(\mathbf{k}, \mathbf{p}^{\prime}, \omega\right)= \\
=\left\{\frac{1}{m}\left(\mathbf{p}+\frac{\hbar \mathbf{k}}{2}\right) \frac{e}{c} \mathbf{A}(\mathbf{k}, \omega)-e \varphi(\mathbf{k}, \omega)\right\} \mathcal{H}\left(\mathbf{k}, \mathbf{p}+\frac{\hbar \mathbf{k}}{2}, \omega\right),
\end{gathered}
$$

где

$$
\begin{aligned}
& \mathcal{H}(\mathbf{k}, \mathbf{p}, \omega)=\frac{1}{\hbar} \frac{F_{0}\left(\mathbf{p}+\frac{\hbar \mathbf{k}}{2}\right)-F_{0}\left(\mathbf{p}-\frac{\hbar \mathbf{k}}{2}\right)}{\omega-\Sigma(\mathbf{k}, \mathbf{p})} \\
\Sigma(\mathbf{k}, \mathbf{p})= & \frac{\mathbf{k p}}{m}-(2 \pi \hbar)^{3} \int \frac{d \mathbf{k}^{\prime}}{(2 \pi)^{3}} \Phi\left(\mathbf{k}^{\prime}\right) \frac{1}{\hbar}\left(F_{0}\left(\mathbf{p}+\hbar \mathbf{k}^{\prime}+\frac{\hbar \mathbf{k}}{2}\right)-\right. \\
& \left.-F_{0}\left(\mathbf{p}-\hbar \mathbf{k}^{\prime}-\frac{\hbar \mathbf{k}}{2}\right)\right) .
\end{aligned}
$$

Решение уравнения (20) можно представить в виде

$$
\begin{aligned}
\delta f(\mathbf{k}, \mathbf{p}, \omega)= & \int d \mathbf{p}^{\prime} \mathcal{F}\left(\mathbf{k}, \mathbf{p}+\frac{\hbar \mathbf{k}}{2}, \mathbf{p}^{\prime}, \omega\right) \times \\
& \times\left\{\frac{\mathbf{p}^{\prime}}{m} \frac{e}{c} \mathbf{A}(\mathbf{k}, \omega)-e \varphi(\mathbf{k}, \omega)\right\} \mathcal{H}\left(\mathbf{k}, \mathbf{p}^{\prime}, \omega\right),
\end{aligned}
$$

где $\mathcal{F}\left(\mathbf{k}, \mathbf{p}, \mathbf{p}^{\prime}, \omega\right)$ - оператор, удовлетворяюший уравнению

$$
\mathcal{F}\left(\mathbf{k}, \mathbf{p}, \mathbf{p}^{\prime}, \omega\right)=\delta\left(\mathbf{p}-\mathbf{p}^{\prime}\right)+\mathcal{H}(\mathbf{k}, \mathbf{p}, \omega) \int d \mathbf{p}^{\prime \prime} \Phi\left(\frac{\mathbf{p}-\mathbf{p}^{\prime \prime}}{\hbar}\right) \mathcal{F}\left(\mathbf{k}, \mathbf{p}^{\prime \prime}, \mathbf{p}^{\prime}, \omega\right) .
$$


Поэтому плотность электрического тока равна

$$
\begin{aligned}
\delta j^{\alpha}(\mathbf{k}, \omega)= & -\frac{e^{2} n_{e}}{m c} A^{\alpha}(\mathbf{k}, \omega)+\frac{e}{m} \int d \mathbf{p} \int d \mathbf{p}^{\prime} p^{\alpha} \mathcal{F}\left(\mathbf{k}, \mathbf{p}, \mathbf{p}^{\prime}, \omega\right) \times \\
& \times\left\{\frac{\mathbf{p}^{\prime}}{m} \frac{e}{c} \mathbf{A}(\mathbf{k}, \omega)-e \varphi(\mathbf{k}, \omega)\right\} \mathcal{H}\left(\mathbf{k}, \mathbf{p}^{\prime}, \omega\right) .
\end{aligned}
$$

После несложных, но громоздких преобразований отсюда можно получить закон Ома в виде

$$
\delta j^{\alpha}(\mathbf{k}, \omega)=\sigma^{\alpha \beta}(\mathbf{k}, \omega) E^{\beta}(\mathbf{k}, \omega)
$$

где

$$
\begin{aligned}
\sigma^{\alpha \beta}(\mathbf{k}, \omega)= & \frac{i e^{2} n_{e}}{m \omega} \delta^{\alpha \beta}-\frac{i e^{2}}{m \omega} \int d \mathbf{p} \int d \mathbf{p}^{\prime} \frac{p^{\alpha} p^{\prime \beta}}{m} \mathcal{F}\left(\mathbf{k}, \mathbf{p}, \mathbf{p}^{\prime}, \omega\right) \times \\
& \times \frac{1}{\hbar} \frac{F_{0}\left(\mathbf{p}^{\prime}+\frac{\hbar \mathbf{k}}{2}\right)-F_{0}\left(\mathbf{p}^{\prime}-\frac{\hbar \mathbf{k}}{2}\right)}{\omega-\Sigma\left(\mathbf{k}, \mathbf{p}^{\prime}\right)}
\end{aligned}
$$

- тензор проводимости. Обшее выражение для тензора диэлектрической проницаемости теперь можно установить на основании соотношения

$$
\varepsilon^{\alpha \beta}(\mathbf{k}, \omega)=\delta^{\alpha \beta}+\frac{4 \pi i}{\omega} \sigma^{\alpha \beta}(\mathbf{k}, \omega)
$$

Полная информация о дисперсионных свойствах однородных изотропных квантовых электронных систем содержится в формулах для продольной $\varepsilon^{1}(\mathbf{k}, \omega)$ и поперечной $\varepsilon^{\operatorname{tr}}(\mathbf{k}, \omega)$ компонентами диэлектрической проницаемости. Для $\varepsilon^{1}(\mathbf{k}, \omega)$ находим

$$
\begin{aligned}
\varepsilon^{l}(\mathbf{k}, \omega)=1 & -\frac{\omega_{p}^{2}}{\omega^{2}}+\frac{4 \pi e^{2}}{m \omega^{2}} \int d \mathbf{p} \int d \mathbf{p}^{\prime} \frac{1}{m} \frac{(\mathbf{k} \mathbf{p})\left(\mathbf{k} \mathbf{p}^{\prime}\right)}{k^{2}} \mathcal{F}\left(\mathbf{k}, \mathbf{p}, \mathbf{p}^{\prime}, \omega\right) \times \\
& \times \frac{1}{\hbar} \frac{F_{0}\left(\mathbf{p}^{\prime}+\frac{\hbar \mathbf{k}}{2}\right)-F_{0}\left(\mathbf{p}^{\prime}-\frac{\hbar \mathbf{k}}{2}\right)}{\omega-\Sigma\left(\mathbf{k}, \mathbf{p}^{\prime}\right)} .
\end{aligned}
$$

Поперечная компонента оказывается равной

$$
\begin{aligned}
\varepsilon^{\operatorname{tr}}(\mathbf{k}, \omega)=1 & -\frac{\omega_{p}^{2}}{\omega^{2}}+\frac{4 \pi e^{2}}{m \omega^{2}} \int d \mathbf{p} \int d \mathbf{p}^{\prime} \frac{1}{2} \frac{\left(\mathbf{p}_{\perp} \mathbf{p}_{\perp}^{\prime}\right)}{m} \mathcal{F}\left(\mathbf{k}, \mathbf{p}, \mathbf{p}^{\prime}, \omega\right) \times \\
& \times \frac{1}{\hbar} \frac{F_{0}\left(\mathbf{p}^{\prime}+\frac{\hbar \mathbf{k}}{2}\right)-F_{0}\left(\mathbf{p}^{\prime}-\frac{\hbar \mathbf{k}}{2}\right)}{\omega-\Sigma\left(\mathbf{k}, \mathbf{p}^{\prime}\right)}
\end{aligned}
$$

где

$$
p_{\perp}^{\alpha}=\left(\delta^{\alpha \beta}-\frac{k^{\alpha} k^{\beta}}{k^{2}}\right) p^{\beta}
$$


Оператор $\mathcal{F}\left(\mathbf{k}, \mathbf{p}, \mathbf{p}^{\prime}, \omega\right)$, содержашийся в формулах (26)-(28), является решением интегрального уравнения (23). При наличии малого параметра он может быть представлен в виде ряда:

$$
\begin{aligned}
& \mathcal{F}\left(\mathbf{k}, \mathbf{p}, \mathbf{p}^{\prime}, \omega\right)=\delta\left(\mathbf{p}-\mathbf{p}^{\prime}\right)+\mathcal{H}(\mathbf{k}, \mathbf{p}, \omega) \Phi\left(\frac{\mathbf{p}-\mathbf{p}^{\prime}}{\hbar}\right)+ \\
& \quad+\mathcal{H}(\mathbf{k}, \mathbf{p}, \omega) \int d \mathbf{p} \Phi\left(\frac{\mathbf{p}-\mathbf{p}^{\prime \prime}}{\hbar}\right) \mathcal{H}\left(\mathbf{k}, \mathbf{p}^{\prime \prime}, \omega\right) \Phi\left(\frac{\mathbf{p}^{\prime \prime}-\mathbf{p}^{\prime}}{\hbar}\right)+ \\
& \quad+\mathcal{H}(\mathbf{k}, \mathbf{p}, \omega) \int d \mathbf{p} \Phi\left(\frac{\mathbf{p}-\mathbf{p}^{\prime \prime}}{\hbar}\right) \mathcal{H}\left(\mathbf{k}, \mathbf{p}^{\prime \prime}, \omega\right) \Phi\left(\frac{\mathbf{p}^{\prime \prime}-\mathbf{p}^{\prime \prime \prime}}{\hbar}\right) \times \\
& \quad \times \mathcal{H}\left(\mathbf{k}, \mathbf{p}^{\prime \prime \prime}, \omega\right) \Phi\left(\frac{\mathbf{p}^{\prime \prime \prime}-\mathbf{p}^{\prime}}{\hbar}\right)+\cdots .
\end{aligned}
$$

Полюсы подынтегральных выражений в формулах (27) и (28) определяются уравнением

$$
\omega-\Sigma(\mathbf{k}, \mathbf{p})=0
$$

где

$$
\begin{aligned}
\Sigma(\mathbf{k}, \mathbf{p}) & =\frac{\mathbf{k p}}{m}-C\left(\mathbf{p}+\frac{\hbar \mathbf{k}}{2}\right)+C\left(\mathbf{p}-\frac{\hbar \mathbf{k}}{2}\right) \\
C(\mathbf{p}) & =\frac{2 e^{2} m}{\pi \hbar^{2} \beta} \frac{1}{p} \int_{0}^{\infty} \frac{d x}{x} \ln \frac{1+e^{\beta \mu} e^{-\beta \frac{p^{2}}{2 m}(x-1)^{2}}}{1+e^{\beta \mu} e^{-\beta \frac{p^{2}}{2 m}(x+1)^{2}}},
\end{aligned}
$$

$\beta=1 / \theta$ - обратная температура. Контур интегрирования по $\omega$ обходит такого рода особенность сверху [12]. В случае полностью вырожденного ферми-газа

$$
\begin{aligned}
C_{\lambda}(p)= & \frac{e^{2}}{2 \pi \hbar^{2}} \frac{p_{\mathrm{F}}^{2}-p^{2}+\lambda^{2}}{p} \ln \frac{\left(p_{\mathrm{F}}+p\right)^{2}+\lambda^{2}}{\left(p_{\mathrm{F}}-p\right)^{2}+\lambda^{2}}+\frac{2 e^{2}}{\pi \hbar^{2}} p_{\mathrm{F}}- \\
& -\lambda \frac{2 e^{2}}{\pi \hbar^{2}}\left\{\operatorname{arctg}\left(\frac{p_{\mathrm{F}}+p}{\lambda}\right)+\operatorname{arctg}\left(\frac{p_{\mathrm{F}}-p}{\lambda}\right)\right\}
\end{aligned}
$$

где $p_{\mathrm{F}}$ - импульс частицы на поверхности Ферми, а параметр $\lambda$ введен таким образом, чтобы точки ветвления функции $C_{\lambda}(p)$, определяемые уравнениями $\left(p_{\mathrm{F}} \pm p\right)^{2}+\lambda^{2}=0$, лежали в области комплексных значений.

Остается указать правила обхода особых точек в формулах (27) и (28) при интегрировании по $p_{\|}=(\mathbf{k p}) / k$. С целью сокращения записи заметим, что в этом случае мы будем иметь дело с интегралами вида

$$
I\left(p_{\perp}, k, \omega\right)=\int_{-\infty}^{+\infty} d p_{\|} \frac{A\left(p_{\perp}, p_{\|}, k\right)}{\omega+i \varepsilon-\Sigma\left(p_{\perp}, p_{\|}, k\right)}
$$

где

$$
\Sigma\left(p_{\perp}, p_{\|}, k\right) \equiv \frac{k}{m} p_{\|}-C\left(p_{\perp}, p_{\|}+\frac{\hbar k}{2}\right)+C\left(p_{\perp}, p_{\|}-\frac{\hbar k}{2}\right)
$$

3 Теоретическая и математическая физика, т. 131, № 2, 2002 г. 
Если $p_{\|}^{(i)}\left(p_{\perp}, k, \omega\right)$ - действительные решения уравнения $\omega-\Sigma\left(p_{\perp}, p_{\|}, k\right)=0$ и эти точки являются полюсами порядка $m_{i}$, то интеграл (33) приводится к виду

$$
\begin{aligned}
& I\left(p_{\perp}, k, \omega\right)=\mathcal{P} \int_{-\infty}^{+\infty} d p_{\|} \frac{A\left(p_{\perp}, p_{\|}, k\right)}{\omega-\Sigma\left(p_{\perp}, p_{\|}, k\right)}- \\
& \quad-\left.i \pi \sum_{i} m_{i}\left|\frac{\partial^{m_{i} \Sigma\left(p_{\perp}, p_{\|}, k\right)}}{\partial^{m_{i}} p_{\|}}\right|^{-1} \frac{\partial^{m_{i}-1} A\left(p_{\perp}, p_{\|}, k\right)}{\partial^{m_{i}-1} p_{\|}}\right|_{p_{\|}=p_{\|}^{(i)}\left(p_{\perp}, k, \omega\right)}
\end{aligned}
$$

Дисперсионные соотношения $\omega=\omega(\mathbf{k})$ для собственных мод квантовой плазмы определяются дисперсионным уравнением [12].

Формулы (26)-(28) являются более обшими, чем те, которые обычно используются для расчетов волновых процессов в квантовой плазме [13]. Эти формулы учитывают обменное кулоновское взаимодействие $(\Sigma(\mathbf{k}, \mathbf{p})$ и ряд $(29)$ начиная со второго члена). Для не слишком больших концентраций электронов достаточно в выражениях (27) и (28) учесть только первый член разложения $(29)$ и положить $\Sigma(\mathbf{k}, \mathbf{p})=\mathbf{k p} / m$, так что влияние обменных корреляций на свойства разреженного электронного газа становится несушественным. Однако для плотной плазмы этого приближения недостаточно. Из формул (26)-(28) непосредственно видно, что обменные кулоновские корреляции приводят, в частности, к сдвигу резонансных частот, величина которого зависит от температуры и концентрации заряженных частиц.

\section{6. ЗАКЛЮЧЕНИЕ}

Таким образом, мы показали, что сформулированная вьше задача об отыскании функции распределения $F(\mathbf{r}, \mathbf{p}, t)$, приводящая бы в результате вычисления статистических средних (математических ожиданий) к тем же локальным значениям фундаментальных физических характеристик системы частиц (числа частиц, импульса и энергии), что и квантовая механика, действительно имеет решение. Сушественно, что такое решение может быть получено в рамках метода, в основе которого лежит лишш квантово-механическое определение плотности вероятности, не ограниченное числом частиц в системе.

Найденная микроскопическая квантовая функция распределения отличается от функции Вигнера, но совпадает с ней в частном случае пространственно-однородных систем.

В уравнении для микроскопической одночастичной функции распределения автоматически появляется двухчастичная функция распределения и т.д., так что мы получаем цепочку уравнений Боголюбова и уравнение Лиувилля для квантовых функций распределения при любом числе частиц в системе.

В рамках такой теории с использованием процедуры группового разложения получено кинетическое уравнение с самосогласованным электромагнитным полем, на основании которого найдены общие формулы для тензора диэлектрической проницаемости, продольной и поперечной составляющих диэлектрической проницаемости электронной компоненты плазмы, а также проанализирована проблема обхода полюсов в интегралах 
при вычислении этих физических характеристик. Наряду с известными физическими эффектами, определяющими дисперсию продольных и поперечных волн в плазме, эти формулы учитывают обменные кулоновские взаимодействия, сушественные для плотных систем. В дисперсионные уравнения для волн вклад обменных корреляций входит в виде ряда, так что для разреженного электронного газа достаточно учесть только первые два члена этого ряда. Без учета обменных кулоновских корреляций найденные выражения совпадают с хорошо известными результатами [8], [13].

Благодарности. С.Г. М. выражает глубокую благодарность Consejo Nacional de Ciencia y Tecnología de México (CONACYT) за поддержку, а также Instituto Tecnológico de Morelia за гостеприимство.

\section{Список литературы}

[1] Л. С. Кузьменков, С. Г. Максимов. ТМФ. 1999. Т. 118. № 2. С. 287.

[2] Л. С. Кузьменков, С.Г. Максимов, В. В. Федосеев. Изв. вузов. Физика. 2000. Т. 43. № 9. C. 8.

[3] Л.С. Кузьменков, С.Г. Максимов, В. В. Федосеев. Вест. Моск. ун-та. Сер. 3. Физика. Астрономия. 2000. № 5. С. 3 .

[4] Л. С. Кузьменков, С.Г. Максимов, В. В. Федосеев. ТМФ. 2001. Т. 126. № 1. С. 136.

[5] Л.С. Кузьменков, С.Г. Максимов, В. В. Федосеев. ТМФ. 2001. Т. 126. № 2. С. 258.

[6] Л. С. Кузьменков. ТМФ. 1991. Т. 86. № 2. С. 231.

[7] К. П. Гуров. Основания кинетической теории. М.: Наука, 1966.

[8] В. П. Силин. Введение в кинетическую теорию газов. М.: Наука, 1971.

[9] Н. Н. Боголюбов. Избранные труды в трех томах. Т. 3. Киев: Наукова думка, 1971.

[10] Н. Н. Боголюбов, Н. Н. Боголюбов (мл.). Введение в квантовую статистическую механику. М.: Наука, 1984.

[11] А. И. Ахиезер, С. В. Пелетминский. Методы статистической физики. М.: Наука, 1977.

[12] А. И. Ахиезер и др. Электродинамика плазмы. М.: Наука, 1974.

[13] А. Ф. Александров, Л. С. Богданкевич, А. А. Рухадзе. Основы электродинамики плазмы. М.: Высшая школа, 1988. после доработки 16.Х.2001 г. 